\title{
Neurofibromatosis type 1: More than skin deep
}

\section{Dermatologic findings manifest in childhood, but the disease progresses to multiple organ systems. Here's how to proceed if you diagnose this in your patient.}

$\mathrm{N}$ eurofibromatosis type 1 (NF1) is an autosomal dominant inherited disorder that is estimated to occur in 1:2500 births and to have a prevalence of 1:2000 to 1:4000., It was first described in 1882 by Friedrich Daniel Von Recklinghausen, who identified patients and their relatives with signs of neuroectodermal abnormalities (café-au-lait macules [CALMs], axillary and inguinal freckling, and neurofibromas).

NF1 may begin insidiously in childhood and evolves as the patient ages. It is associated with intracranial, intraspinal, and intraorbital neoplasms, although other organs and tissues can also be involved.

The family physician might be the first one to recognize the signs of this condition during a well-child exam and is in a unique position to coordinate a multidisciplinary approach to care.

\section{A mutated allele and early manifestations on the skin}

NF1 has been attributed to genetic mosaicism and is classified as segmental, generalized, or (less frequently) gonadal. The disorder results from germline mutations in the NF1 tumorsuppressor gene on chromosome 17 , known to codify the cytoplasmic protein called neurofibromin. ${ }^{3}$ The penetrance of NF1 is complete, which means that $100 \%$ of patients with the mutated allele will develop the disease.

Patients typically have symptoms by the third decade of life, although many will show signs of the disease in early childhood. CALMs are the earliest expression of NF1. They manifest in the first 2 years of life and are found in almost all affected patients. The lesions are well defined and measure 10 to $40 \mathrm{~mm}$. They are typically light brown, although they may darken with sun exposure.

Histologically, the lesions will show macromelanosomes and high concentrations of melanin but do not represent an increased risk for malignancy. ${ }^{4}$ Not all isolated CALMs are a sign of NF1. While children younger than 29 months with 6 or more CALMs have a high risk for NF1 (80.4\%; 95\% confidence interval [CI], $74.6 \%$ to $86.2 \%$ ), those who are older than 29 months with at least 1 atypical CALM or fewer than 6 CALMs have just a $0.9 \%$ (95\% CI, $0 \%$ to $2.6 \%$ ) risk for constitutional NF1. ${ }^{5}$

Freckles are also observed in $90 \%$ of patients with NFl; these tend to develop after the third year of life. The breast and trunk are the most commonly affected areas in adults. The pathophysiology is unknown, but this freckling is believed to be related to skin friction, high humidity, and ambient temperature. ${ }^{6}$

Neurofibromas are benign subcutaneous palpable lesions that grow within peripheral nerve tissue, including spinal, subcutaneous, plexiform, or dermal encapsulated nerves. Originating in Schwann cells, they are composed of fibroblasts, mast cells, macrophages, endothelial cells, and other perineural cells. Some patients show disfiguration when hundreds of these masses are
T. Grant Phillips, MD; Osiris R. Persia, MD; Jolenny A. Jimenez Lopez, MD UPMC Altoona Family Physicians Residency, PA (Dr. Phillips); Department of Internal Medicine, BronxCare Health System, NY (Dr. Persia); Department of Internal Medicine (Radiology), Hospital Metropolitano de Santiago, Dominican Republic (Dr. Jimenez Lopez)

\section{-Dphillipstg2@upmc.edu}

The authors reported no potential conflict of interest relevant to this article.

doi: $10.12788 / j$ fp. 0082 


\section{$>$}

\section{Some}

patients show disfiguration when hundreds of neurofibromas are present. present (FIGURE). These tumors increase in number as the patient ages or during pregnancy, which is thought to be secondary to hormonal changes. ${ }^{7}$ They are sometimes painful and can be pruritic. Their appearance can also cause patient distress.

\section{The diagnosis is a clinical one}

Suspicion for NF1 should be high in patients presenting with the dermatologic findings described, although CALMs and freckling are not exclusive to NF1. Diagnostic criteria for NF1, which distinguish it from other conditions, were first outlined in a National Institutes of Health Consensus Development Conference Statement in $1987 .{ }^{8}$ The list of criteria has subsequently been expanded.

While the presence of at least 2 criteria is required for diagnosis, ${ }^{2} \mathrm{NF} 1$ should be suspected in individuals who have any of the following findings $\mathrm{s}^{8,9}$ :

- the presence of at least 6 CALMs that are $>5 \mathrm{~mm}$ in prepubertal children and $>15 \mathrm{~mm}$ in adults

- 2 or more neurofibromas of any type, or at least one plexiform neurofibroma

- axillary or groin freckling

- optic pathway glioma

- 2 or more Lisch nodules (iris hamartomas seen on slit-lamp examination)

- bony dysplasia (sphenoid wing dysplasia, bowing of long bone \pm pseudarthrosis)

- first-degree relative with NF1.

\section{What you'll see}

\section{as the disease progresses}

NF1 can affect a variety of systems, and potential complications of the disease are numerous and varied (see TABLE$^{9}$ ). Here is some of what you may see as the patient's disease progresses to various organ systems:

I Learning disabilities and other cognitive and behavioral problems, such as attention-deficit/hyperactivity disorder, may affect up to $70 \%$ of children with NF1. Additionally, children with NF1 have visual/spatial problems, impaired visual motor integration, and language deficits. ${ }^{10}$ The etiology of cognitive impairment in NF1 is unknown. ${ }^{11}$
I Hypertension is common and may contribute to premature death in patients with NF1. Up to $27 \%$ of patients will have significant cardiovascular anomalies, including pulmonary valve stenosis, hypertrophic cardiomyopathy in patients with complete deletions of the NF1 gene, intracardiac neurofibromas, renal artery stenosis, coarctation of the aorta, and cerebral infarctions. ${ }^{12}$ Renal artery stenosis occurs in approximately $2 \%$ of the NF1 population, and the diagnosis should be considered in hypertensive children, young adults, pregnant women, older individuals with refractory hypertension, and those with an abdominal bruit. ${ }^{13}$

I Psychological issues. The disfigurement caused by neurofibromas and the uncertainty of an unpredictable disease course can cause psychological manifestations for patients with NF1. Anxiety and depression are common. Not surprisingly, patients with more severe disease report more adverse psychological effects.

I Orthopedic deformities. Spinal deformities are the most common skeletal manifestation of NF1, with an incidence estimated from $10 \%$ to $25 \%$ in various studies. Bone mineral density, as measured by age- and gender-adjusted Z-scores, is significantly lower in NF1 patients than in the general population. ${ }^{14}$ Children may develop bowing of the long bones, particularly the tibia, and pseudarthrosis, a false joint in a long bone. Children with NF1 need yearly assessment of the spine. Patients with clinical evidence of scoliosis should be referred to Orthopedics for further evaluation.

I Eye issues. A majority of adult patients develop neurofibroma-like nodules in the iris known as Lisch nodules. The nodules are not thought to cause any ophthalmologic complications. Patients may also develop palpebral neurofibroma, which may become large and sporadically show malignant transformation. Optic nerve glioma may cause strabismus and proptosis, and a large number of patients will also develop glaucoma and globe enlargement. ${ }^{15}$

I Gastrointestinal lesions and cancer. Neurofibromas can grow in the stomach, liver, mesentery, retroperitoneum, and bowel. Adenocarcinoma developed in $23 \%$ 


\section{FIGURE}

\section{Patients may experience disfigurement when hundreds} of neurofibromas are present
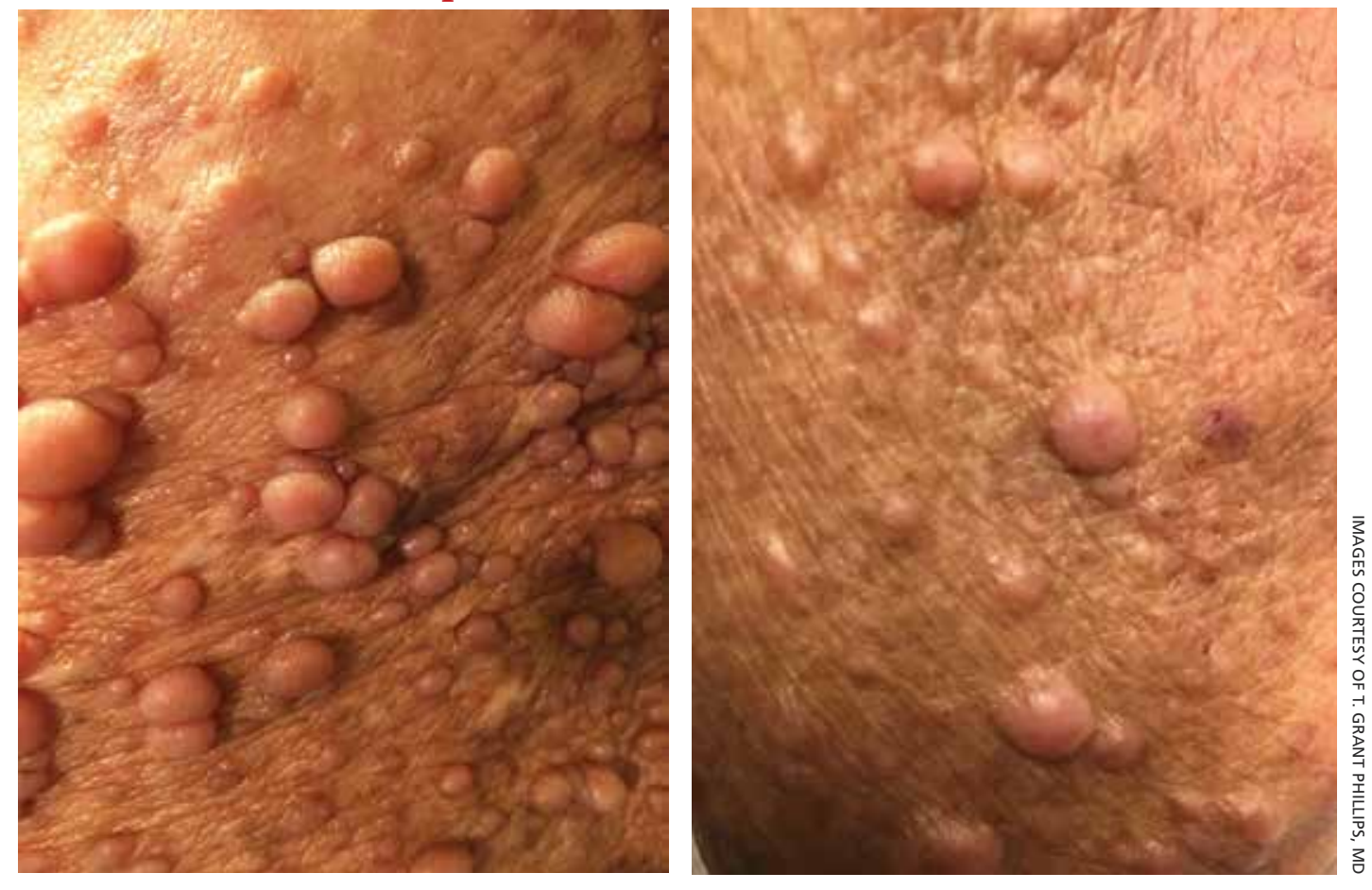

of patients. ${ }^{16}$ Gastrointestinal tract bleeding, pseudo-obstruction, and protein-losing enteropathy also may occur. ${ }^{17}$

I Central nervous system manifestations. Neurological manifestations have been observed in $55 \%$ of patients with NF1. ${ }^{18}$ These include headache, hydrocephalus, epilepsy, lacunar stroke, white matter disease, intraspinal neurofibroma, facial palsy, radiculopathy, and polyneuropathy. Tumors include optic pathway tumors, meningioma, and cerebral glioma. Glioma is the predominant tumor type in NF1 and occurs in all parts of the nervous system, with a predilection for the optic pathways, brainstem, and cerebellum. ${ }^{18}$

I Malignant peripheral nerve sheath tumors. There is an $8 \%$ to $13 \%$ lifetime risk for malignant peripheral nerve sheath tumors (MPNST), predominantly in individuals between the ages of 20 and 35. ${ }^{19,20}$ Any change in neurofibroma from soft to hard, or a rapid increase in the size, is suspicious for MPNST. Other symptoms include persistent pain lasting for longer than a month, pain that disturbs sleep, and new neurological deficits. These cancers can be hard to detect, leading to poor prognosis secondary to metastasis. $^{19,20}$ The greatest risk factors for MPNST are pain associated with a mass and the presence of cutaneous and subcutaneous neurofibromas. ${ }^{21}$

\section{Treatment is symptom based, but there is a new option}

Treatment is individualized to the patient's symptoms. Neurofibromas that are disfiguring, disruptive, or malignant may be surgically removed.

In April 2020, the US Food and Drug Administration (FDA) approved selumetinib (Koselugo) for the treatment of pediatric patients (ages $\geq 2$ years) with NF1 who have symptomatic, inoperable plexiform neurofibromas (PNs). ${ }^{22}$ In a clinical trial, patients received selumetinib $25 \mathrm{mg} / \mathrm{m}^{2}$ orally twice a day until they demonstrated disease progression or experienced "unacceptable" adverse 
In light of the condition's heterogeneity, the goals of care include early recognition and treatment of complications, especially neoplasms.

TABLE

Common clinical manifestations of neurofibromatosis ${ }^{9}$

\begin{tabular}{l|l|l}
\hline Finding & Rate of occurrence & Age at onset \\
\hline Café-au-lait macules & $>99 \%$ & Birth to $12 \mathrm{y}$ \\
\hline Cutaneous neurofibromas & $>99 \%$ & $>7 \mathrm{y}$ \\
\hline Lisch nodules & $90 \%-95 \%$ & $>7 \mathrm{y}$ \\
\hline Learning problems & $30 \%-60 \%$ & Birth \\
\hline Plexiform neurofibromas & $30 \%-50 \%$ & Birth to $18 \mathrm{y}$ \\
\hline Optic pathway glioma & $15 \%$ & Birth to $7 \mathrm{y}$ \\
\hline Scoliosis & $10 \%$ & Birth to $18 \mathrm{y}$ \\
\hline Severe cognitive impairment (IQ $<70)$ & $4 \%-8 \%$ & Birth \\
\hline Epilepsy & $6 \%-7 \%$ & Lifelong \\
\hline Disfiguring facial plexiform neurofibromas & $3 \%-5 \%$ & Birth to $5 \mathrm{y}$ \\
\hline Malignant peripheral nerve sheath tumor & $2 \%-5 \%$ & $5-75 \mathrm{y}$ \\
\hline Cerebral gliomas & $2 \%-3 \%$ & Lifelong \\
\hline
\end{tabular}

events. ${ }^{22,23}$ The overall response rate was $66 \%$, defined as "the percentage of patients with a complete response and those who experienced more than a $20 \%$ reduction in $\mathrm{PN}$ volume on MRI that was confirmed on a subsequent MRI within 3 to 6 months." ${ }^{22}$

Of note, all patients had a partial, not complete, response. Common adverse effects included vomiting, rash, abdominal pain, diarrhea, and nausea. ${ }^{23}$ Selumetinib may also cause more serious adverse effects, including cardiomyopathy and ocular toxicity. Prior to treatment initiation and at regular intervals during treatment, patients should undergo cardiac and ophthalmic evaluation. ${ }^{22,23}$ Selumetinib was granted priority review and orphan drug status by the FDA.22

\section{You play a key role}

\section{in ongoing monitoring}

In light of the condition's heterogeneity, the goals of care include early recognition and treatment of complications, especially neoplasms; optimization of quality of life; and identification and treatment of comorbidities. Family physicians are well positioned to monitor patients with NF1 for age-specific disease manifestations and potential complications. ${ }^{9}$ All patients require:

- an annual physical examination by a physician who is familiar with the individual and with the disease

- annual ophthalmologic examination in early childhood; less frequent examination in older children and adults

- regular blood pressure monitoring

- other studies (eg, MRI) only as indicated on the basis of clinically apparent signs or symptoms

- monitoring by an appropriate specialist if there are abnormalities of the central nervous, skeletal, or cardiovascular systems

- referral to a neurologist for any unexplained neurological signs and symptoms. Referral should be urgent if there are acute symptoms of progressive sensory disturbance, motor deficit and incoordination, or sphincter disturbances since these might indicate an intracranial lesion or spinal cord compression. Headaches on waking, morning vomiting, and altered consciousness are suggestive of raised intracranial pressure.

I Children with NF1 benefit from coordinated care between the FP and a pediatrician or other specialist familiar with the disease. In addition to providing usual well care, perform regular assessment of development and 
school performance. Pay careful attention to the cardiovascular system (particularly blood pressure) and evaluate for scoliosis.

I Young adults should be continually monitored for all complications, especially hypertension. This population requires continued education about NF1 and its possible complications and may benefit from counseling about disease inheritance. Screen for anxiety and depression; offer psychological support.

I Adults require monitoring based on patient preference and disease severity. For this population, blood pressure should be measured annually, or more frequently if the patient's values indicate borderline hypertension. Provide education about complications, especially MPNSTs and spinal cord compression. Patients who have abnormalities of the central nervous, skeletal, or cardiovascular systems should be monitored by an appropriate specialist. If desired, the patient may be referred to a geneticist, especially if he or she expresses concern about inheritance. Cutaneous neurofibromas can be removed if they cause discomfort, although removal occasionally results in neurological deficit. JFP

CORRESPONDENCE

T. Grant Phillips, MD, Associate Director, UPMC Altoona Family Physicians Residency, 501 Howard Avenue, Altoona, PA 16601-4899; phillipstg2@upmc.edu

\section{REFERENCES}

1. Ly KI, Blakeley JO. The diagnosis and management of neurofibromatosis type 1. Med Clin North Am. 2019;103:1035-1054.

2. Miller DT, Freedenberg D, Schorry E, et al; Council on Genetics, American College of Medical Genetics and Genomics. Health supervision for children with neurofibromatosis type 1. Pediatrics. 2019;143:e20190660.

3. Boyd KP, Korf BR, Theos A. Neurofibromatosis type 1. J Am Acad Dermatol. 2001;61:1-14.

4. Hirbe AC, Gutmann DH. Neurofibromatosis type 1: a multidisciplinary approach to care. Lancet Neurol. 2014;13:834-844.

5. Ben-Shachar S, Dubov T, Toledano-Alhadef H, et al. Predicting neurofibromatosis type 1 risk among children with isolated caféau-lait macules. J Am Acad Dermatol. 2017;76:1077-1083.e3.

6. Friedman JM. Neurofibromatosis 1. In: Adam MP, Ardinger $\mathrm{HH}$ Pagon RA, et al, eds. GeneReviews' [Internet]. Seattle (WA): University of Washington, Seattle; 1993-2020. www.ncbi.nlm.nih. gov/books/NBK1109. Accessed Septemeber 28, 2020.

7. Roth TM, Petty EM, Barald KF. The role of steroid hormones in the NF1 phenotype: focus on pregnancy. Am J Med Genet A. 2008;146A:1624-1633.

8. National Institutes of Health Consensus Development Conference Statement: neurofibromatosis. Bethesda, MD, July 13-15, 1987. Neurofibromatosis. 1988;1:172-178. https://consensus. nih.gov/1987/1987Neurofibramatosis064html.htm. Accessed Septemeber 28, 2020.

9. Ferner RE, Huson SM, Thomas N, et al. Guidelines for the diagnosis and management of individuals with neurofibromatosis 1 . J Med Genet. 2007;44:81-88.

10. Koth CW, Cutting LE, Denckla MB. The association of neurofibromatosis type 1 and attention deficit hyperactivity disorder. Child Neuropsychol. 2000;6:185-194.

11. North KN, Riccardi VM, Samango-Sprouse C, et al. Cognitive function and academic performance in neurofibromatosis 1 consensus statement from the NF1 Cognitive Disorders Task Force. Neurology. 1997;48:1121-1127.

12. Gerber PA, Antal AS, Neumann NJ, et al. Neurofibromatosis. Eur J Med Res. 2009;14:102-105.

13. Friedman JM, Arbiser J, Epstein JA, et al. Cardiovascular disease in neurofibromatosis 1: a report of the NF1 Cardiovascular Task Force. Genet Med. 2003;4:105-111.

14. Lammert M, Kappler M, Mautner VF, et al. Decreased bone mineral density in patients with neurofibromatosis 1. Osteoporos Int 2005;16:1161-1166.

15. Abdolrahimzadeh B, Piraino DC, Albanese G, et al. Neurofibromatosis: an update of ophthalmic characteristics and applications of optical coherence tomography. Clin Ophthalmol. 2016;10:851-860.

16. Bakker JR, Haber MM, Garcia FU. Gastrointestinal neurofibromatosis: an unusual cause of gastric outlet obstruction. Am Surg. 2005; 71:100-105.

17. Rastogi R. Intra-abdominal manifestations of von Recklinghausen's neurofibromatosis. Saudi J Gastroenterol. 2008;14:80-82.

18. Créange A, Zeller J, Rostaing-Rigattieri S, et al. Neurological complications of neurofibromatosis type 1 in adulthood. Brain. 1999;122(pt 3):473-481

19. Ferner RE, Gutmann DH. International consensus statement on malignant peripheral nerve sheath tumours in neurofibromatosis 1. Cancer Res. 2002;62:1573-1577.

20. Evans DG, Baser ME, McGaughran J, et al. Malignant peripheral nerve sheath tumors in neurofibromatosis $1 . \mathrm{J}$ Med Genet. 2002;39:311-314.

21. King AA, Debaun MR, Riccardi VM, et al. Malignant peripheral nerve sheath tumors in neurofibromatosis 1 . Am J Med Genet. 2000;93:388-392.

22. US Food and Drug Administration. FDA approves first therapy for children with debilitating and disfiguring rare disease [news release]. April 10, 2020, www.fda.gov/news-events/ press-announcements/fda-approves-first-therapy-childrendebilitating-and-disfiguring-rare-disease. Accessed Septembe 28,2020 .

23. Koselugo (selumetinib) [product information]. Wilmington, DC AstraZeneca Pharmaceuticals LP; April 2020. www.accessdata. fda.gov/drugsatfda_docs/label/2020/213756s000lbl.pdf. Accessed September 24, 2020
Provide adult patients with education about complications, especially malignant peripheral nerve sheath tumors and spinal cord compression. 